\title{
STELLAR MAGNETIC IMAGING FROM \\ SPECTROPOLARIMETRIC DATA
}

\section{J.-F. DONATI}

Obs. Midi-Pyrénées, 14 Av. Belin, F-31400 Toulouse, France

\begin{abstract}
In this paper, I will review the capabilities of magnetic imaging (also called Zeeman-Doppler imaging) to reconstruct spot distributions of surface fields from sets of rotationnally modulated Zeeman signatures in circularly polarised spectral lines. I will then outline a new method to measure small amplitude magnetic signals (typically $0.1 \%$ for cool active stars) with very high accuracy. Finally, I will present and comment new magnetic images reconstructed from data collected in 1993 December at the Anglo-Australian Telescope (AAT).
\end{abstract}

\section{Introduction}

The basic principles of stellar magnetic imaging are very similar to those of conventional Doppler imaging. Once more, the idea is to reconstruct a stellar image from sets of rotationnally modulated line profiles, with the help of a maximum-entropy image reconstruction algorithm (see Rice, this conference). The main difference is that we are now using sets of polarised line profiles to recover a vector image of the surface magnetic topology.

In Sect. 2, I will outline the specific aspects of Zeeman-Doppler imaging. Note that I will restrict myself to reconstructions of spot distributions of magnetic fields from sets of circularly polarised profiles, as this is the field where most of the work has concentrated to date (Brown et al. 1991; Donati et al. 1992b). In Sect. 3, I will explain the principles of least-square deconvolution, a new useful tool for high accuracy measurements of Zeeman signatures from all spectral lines simultaneously. As a final illustration of the new capabilities of Zeeman-Doppler imaging, I will briefly comment recent magnetic images derived from 1993 AAT observations. 


\section{Simulated magnetic imaging}

A major advantage of magnetic imaging is that it is considerably less prone to artifacts than conventional Doppler imaging. It has been emphasised many times already in this conference how strongly Doppler imaging is sensitive to errors in assumed stellar parameters (Rice, this conference) or to inaccurate modelling of local line profiles (Unruh, this conference). One can indeed easily understand that any errors in estimating the so-called "blank-star profile" will lead to problems in interpreting deviations from this blank-star profile, and therefore to artifacts in reconstructed maps. In magnetic imaging, the blank-star profile (corresponding to a $\mathbf{B}=0$ image) is perfectly well known; indeed, no field automatically implies null polarised profiles, so that no artifacts are expected with this method.

A first series of simulations (Brown et al. 1991) has already proven that spot distributions could be well recovered from sets of rotationnally modulated Stokes $V$ (circular polarisation) profiles. However, these simulations were restricted to radial field distributions only, reconstructed under a radial field assumption. Although it sounded reasonable an hypothesis at that time, it does no longer look particularly appropriate, especially when referring to more recent results obtained on cool active stars (Donati at al. $1992 \mathrm{~b}$ ) where regions of mainly azimuthal field were detected. New imaging simulations with more complex spot distributions were carried out, pointing out to interesting peculiarities of Zeeman-Doppler imaging.

In the simulation shown in Fig. 1, the original image features altogether six spots of equal magnetic strength $(500 \mathrm{G})$, but different latitudes and vector field orientation. The inclination angle $i$ and projected rotational velocity $v \sin i$ of the model star are set to $33^{\circ}$ and $39 \mathrm{~km} \mathrm{~s}^{-1}$ respectively. Note that this image is nothing but a test image with no claim to represent some kind of real field topology. The synthetic circularly polarised profiles (corresponding to a unit Landé factor line at $550 \mathrm{~nm}$ with a $3 \mathrm{~km} \mathrm{~s}^{-1}$ Doppler width and $90 \%$ central depth gaussian local profile) are computed for 10 evenly-spaced phases. The Stokes $V$ signatures reach a maximum amplitude of about $0.2 \%$, typical to what is observed for cool active stars. The reconstructed distribution corresponds to a unit $\chi^{2}$ maximum entropy fit to the synthetic data, noised at a relative level of $2 \times 10^{-5}$. As shown in Sect. 3, this is typical to what can now achieved with new multiline techniques. Comparable results are obtained for a noise level of $5 \times 10^{-5}$.

Obviously, the code is quite successful in distinguishing azimuthal field regions from all other ones. If radial and meridional field spots are also correctly recovered, some crosstalk is observed between these two field components for low latitude features. This is an important property of ZeemanDoppler imaging, that one should always keep in mind when interpreting 


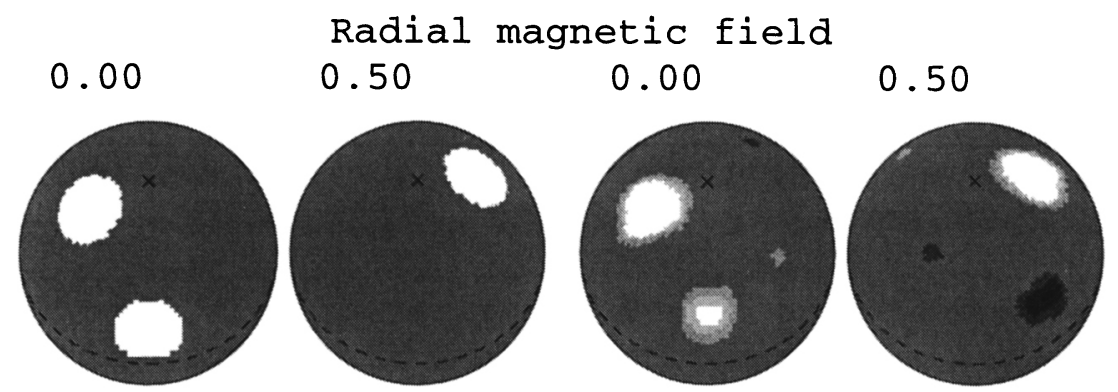

Meridional magnetic field

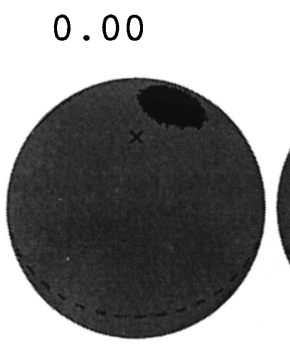

0.50

0.00

0.50
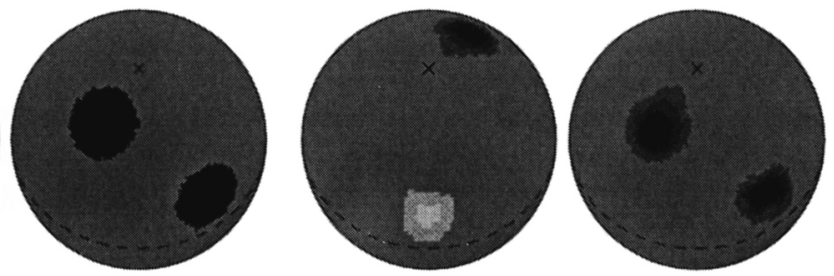

Azimuthal magnetic field

0.00

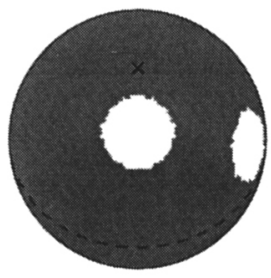

0.50

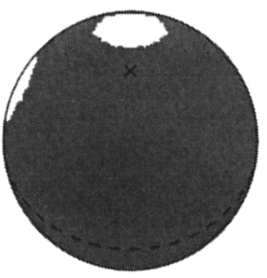

0.00

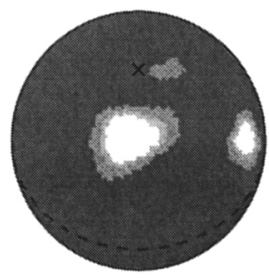

0.50

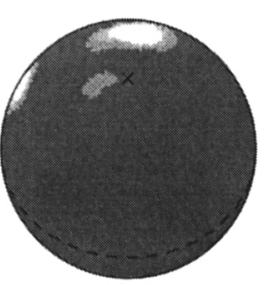

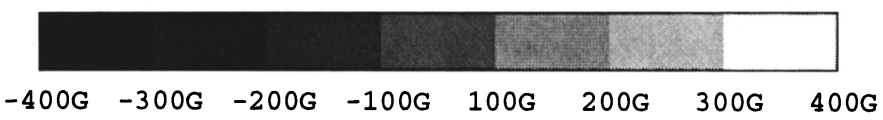

Figure 1. Six-spot star simulation. The original and reconstructed images (two first and two last columns respectively) are shown at phases 0.0 and 0.5 , for each field component

magnetic images from real data. It can be explained by the fact that Stokes $V$ magnetic signatures are only sensitive to the line-of-sight projected (or longitudinal) component of the field vector. Azimuthal field signatures will thus reach a maximum amplitude when the corresponding region gets close to the east and west stellar limbs, and will vanish at the subobserver lon- 
gitude. As the opposite holds for radial and low latitude meridional field regions, the code has some information (through the rotational modulation of Stokes $V$ signatures) to reconstruct to a certain degree the orientation of the field vector in magnetic regions.

Note also that only the upper part of the low latitude radial and azimuthal field spots is reconstructed. In the lower part of these features, the field vector is almost always perpendicular to the line of sight and therefore generates very weak Stokes $V$ signatures. This effect mimics a strong increase in limb darkening, as already evidenced by Brown et al. (1991). Other simulations (not shown here) have also demonstrated that overlapping regions of different magnetic field components are also correctly recovered (to the limits already outlined above), indicating that they do not mutually obscure each other in the reconstruction process. We have noticed as well that the main consequence of moderate phase coverage is essentially to enhance the effects listed above, and in particular radial-to-meridional field crosstalk.

Finally, it is also worth noting that what Zeeman-Doppler imaging measures is magnetic flux (averaged over a resolution element) rather than actual field strength, just as circularly polarised line profiles in the weak field regime do. Moreover, reconstructed fluxes are further affected by surface brightness inhomogeneities. Since what is expected on late-type active stars is cool spots (with a brightness of typically $20 \%$ to $30 \%$ that of the photosphere) with low overall filling factors (likely less than 0.3), it is clear that the field values derived by Zeeman-Doppler imaging can be easily underestimated by an order of magnitude. Inverting simultaneously unpolarised and circularly polarised profiles of infrared lines should be a possible way of fixing this problem in the future, as it would yield a global self-consistent description (temperature, magnetic field and filling factor) of the horizontal structure of stellar atmospheres.

\section{High accuracy measurements of Zeeman signatures}

Zeeman signatures in cool active stars are very small, with typical relative amplitudes of a few tenths of a percent (Donati et al. 1992a). We have seen in Sect. 2 that reconstructing the parent field distribution requires that the shape of such small signatures is measured with high accuracy, i.e. with a $\mathrm{S} / \mathrm{N}$ ratio in Stokes $V$ profiles of at least 40. Clearly, one cannot get such low noise levels (typically $5 \times 10^{-5}$ ) from a single line in a single spectrum (where we get at best noises of $10^{-3}$ ). Moreover, as cool active stars rotate quite rapidly and vary on timescales of a few rotations, one cannot add up more than a few spectra together to decrease the noise level still further. A much more interesting possibility is to extract the polarisation information 
from as many lines as possible in a single spectrum, as all lines are expected to exhibit Zeeman signatures with more or less the same shape.

Assume a given magnetic configuration. In the weak field regime, each Stokes $V$ local line profile obeys:

$$
V_{\mathrm{loc}}(v) \propto g \lambda \frac{\partial I_{\mathrm{loc}}(v)}{\partial v}
$$

where $v$ represents the velocity coordinate $c \Delta \lambda / \lambda$ associated to a wavelength shift $\Delta \lambda$ from line centre wavelength $\lambda$, while $g$ and $I_{\text {loc }}(v)$ are respectively the magnetic sensitivity (Landé factor) and local profile of the selected line. Note that the proportionality factor is the same for all lines and only depends on the local longitudinal field value. If I now further assume that $I_{\text {loc }}(v)$ is similar in shape for all lines and simply scales up in depth with the maximum local line depth $d$, I get:

$$
V_{\mathrm{loc}}(v)=g \lambda d k_{\mathrm{B}}(v)
$$

where $k_{\mathrm{B}}(v)$ is a proportionality function (equal for all lines). In principle, this second approximation is strictly valid only for optically thin lines. However, it is not too bad a model at the scale of a rotationnally broadened line profile (typically tens of $\mathrm{km} \mathrm{s}^{-1}$ ), provided very strong lines (like Balmer or $\mathrm{Ca}$ II $\mathrm{H} \& \mathrm{~K}$ lines) are excluded. Integrating the previous equation over the whole rotating star (i.e. over all points $M$ of brightness and radial velocity $b_{\mathrm{M}}$ and $v_{\mathrm{M}}$ ) gives:

$$
\begin{aligned}
V(v)=\iint b_{\mathrm{M}} V_{\mathrm{loc}}\left(v-v_{\mathrm{M}}\right) d S & =g \lambda d \iint b_{\mathrm{M}} k_{\mathrm{B}}\left(v-v_{\mathrm{M}}\right) d S \\
& =w Z(v)
\end{aligned}
$$

If I finally assume wavelength-independent limb-darkening, the integral function $Z(v)$ - called mean Zeeman signature - is constant for all lines, and its shape is reproduced by all Stokes $V(v)$ profiles with a magnification factor $w=g \lambda d$.

Calling $P(v)$ the line pattern function defined by:

$$
P(v)=\sum_{i} w_{i} \delta\left(v-v_{i}\right)
$$

where $v_{i}$ and $w_{i}$ are respectively the position (in velocity space) and weight of each spectral line, the circularly polarised spectrum $V$ can be written as a convolution expression $(V=P * Z)$ or equivalently as a linear system $(\mathbf{V}=\mathbf{P} \cdot \mathbf{Z})$. Looking for the least-square solution in $\mathbf{Z}$ yields:

$$
\mathbf{Z}=\left({ }^{\mathbf{t}} \mathbf{P} \cdot \mathbf{P}\right)^{-\mathbf{1}}{ }^{\mathbf{t}} \mathbf{P} \cdot \mathbf{V}
$$




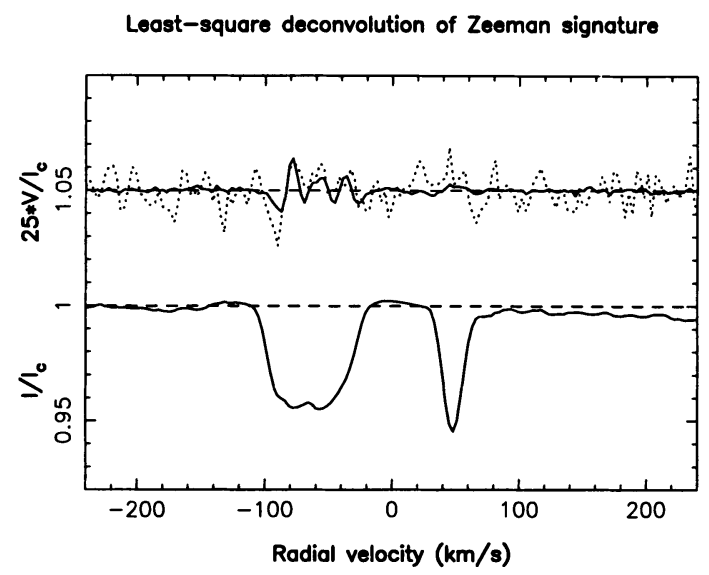

Figure 2. Mean Zeeman signature of the RS CVn system HR 1099 (AAT spectrum). The bottom curve displays the mean unpolarised profile (two absorption lines corresponding to the two system components) while the upper curves present the mean circularly polarised signal derived by least-square deconvolution (full line) and by the old three-line analysis (dotted line). Note that polarised spectra are expanded by 25 and shifted upwards by 1.05 for graphics purposes.

In CORAVEL terminology, ${ }^{\mathbf{t}} \mathbf{P}$ is called line mask while ${ }^{\mathbf{t}} \mathbf{P} \cdot \mathbf{V}$ is the crosscorrelation function, usually taken as the solution. This inversion is slightly more sophisticated in the sense that I "clean" the cross-correlation function from the autocorrelation profile to derive the final solution. I call the overall process least-square deconvolution. Masks have been computed for several model atmospheres ranging from A0 to M0, with the help of Kurucz's atomic database, model atmosphere library and ATLAS9 routines (Kurucz, this conference). The Landé factors are also taken from Kurucz whenever available (i.e. in $90 \%$ cases) and estimated from LS coupling otherwise.

As one can judge from Fig. 2, the technique works remarkably well. More than 1100 lines were used together to derive the mean Zeeman signature on the active RS CVn system HR 1099. A complex magnetic signal is clearly detected in conjunction with the broad line of the primary (most active) star. Compared to the older three-line analysis of Donati et al. (1992a), the sensitivity has increased by about $4.5 \mathrm{mag}$, without which the Stokes $V$ signal could not have been detected (see graph). Similar Zeeman signatures, and thus direct evidence for the presence of magnetic fields, were obtained for the first time ever on two young objects (the PMS system HD 155555 and the ZAMS star LQ Hya) and on five evolved binaries (namely EI Eri, IL Hya, CF Tuc, TY Pyx and SZ Psc). Note that reliability tests on a sharp line object like the Sun (whose integrated light show very little circular polarisation) yield flat Zeeman signatures down to a relative noise level of 
$1.8 \times 10^{-5}$, and give us strong confidence about the reality of our magnetic detections. For HR 1099, Zeeman signatures collected exactly one rotational cycle apart are identical at photon noise accuracy, which demonstrates that the short term variability of magnetic signals on this star is indeed due to rotational modulation.

A second advantage of this multiline technique is that one can very easily correlate Zeeman signatures with various parameters. Their dependence with excitation potential (for a given element and ionisation state, say Fe I) is of particular interest here as it gives us some hints on where the detected magnetic signal comes from. If it is generated in plages (where low excitation $\mathrm{Fe}$ I lines get weaker that high excitation ones), it is expected to increase significantly with excitation potential. A very preliminary result indicates that Zeeman signatures obtained by least-square deconvolution from high excitation $\mathrm{Fe}$ I lines are roughly similar to those derived from low excitation $\mathrm{Fe} \mathrm{I}$ lines (all other parameters kept constant in average). If this is further confirmed, it would suggest that the detected magnetic regions coincide with cool spots rather than plages.

\section{Application to HR 1099}

As an illustrative conclusion for my paper, a very recent (but still preliminary) magnetic map of HR 1099, reconstructed from least-square deconvolved AAT polarised spectra (recorded in 1993 December), is presented in Fig. 3. Magnetic images of HR 1099 (in 1992 December) and LQ Hya (in 1993 December) have also been derived (though not shown here) with the same method. The reconstructed magnetic topology of HR 1099 is significantly more complex than that derived from earlier observations (Donati et al. 1992b). This increase in complexity is also seen in the shape of the polarised signatures themselves and is therefore not attributable to the improved data quality. AAT observations of HR 1099 (collected regularly from 1991) indeed show a progressive evolution (probably linked to some activity cycle) towards more complicated magnetic topologies.

Regions of mostly azimuthal field are detected again on this star in 1992 and 1993, as well as on LQ Hya in 1993. As simulations tend to indicate that such features cannot be interpreted as artifacts (even in case of moderate phase coverage), it looks as if they may be real (although I admit this is very intriguing). Note finally that the reconstructed field strengths are much smaller (typically an order of magnitude) than equipartition values as well as previously published measurements for this kind of objects. Although one must remember that such attenuation factors are indeed expected in Zeeman-Doppler imaging (see Sect. 2), this is indeed a problem that must be worked out carefully in the very near future. 
HR 1099, 1993 December, AAT spectra,

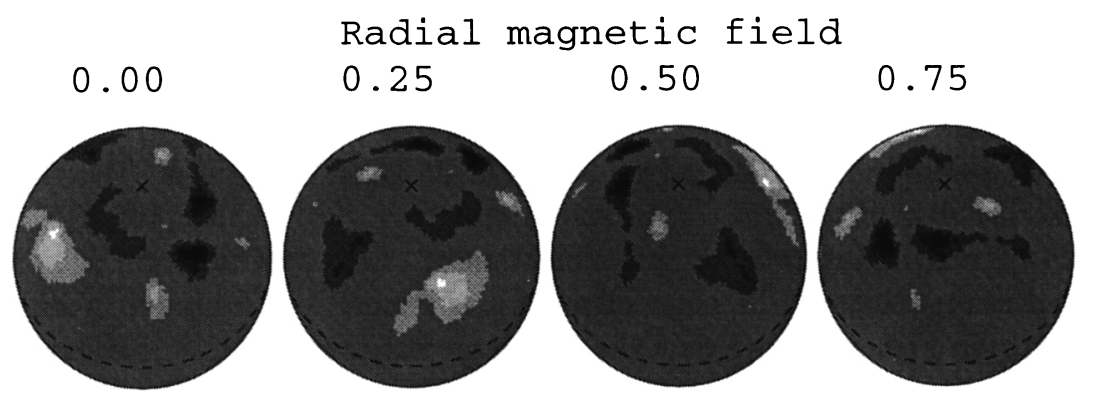

Meridional magnetic field

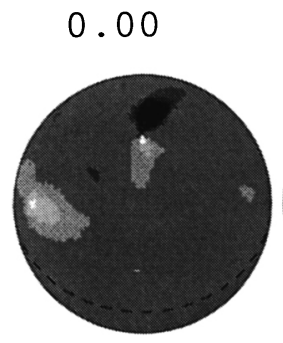

0.25

0.50

0.75
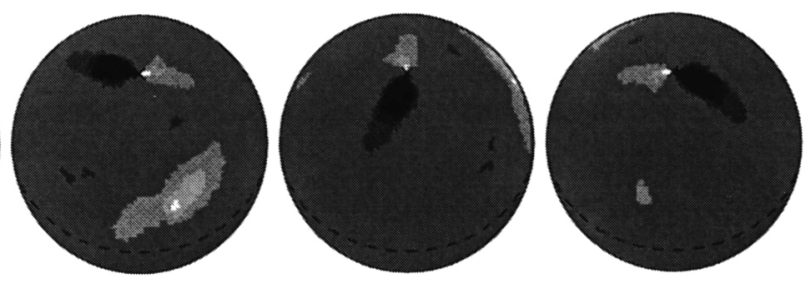

Azimuthal magnetic field

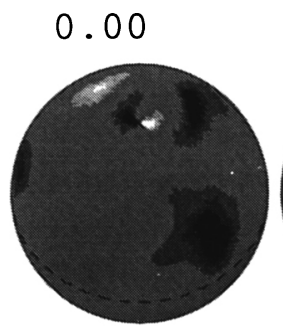

0.25

0.50

0.75

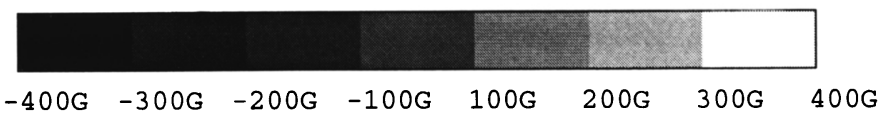

Figure 3. Preliminary magnetic map of HR 1099 in 1993 December

\section{References}

Brown, S.F., Donati, J.-F., Rees, D.E. and Semel, M. (1991) $A \mathscr{E} A, \mathbf{2 5 0}, 463$

Donati, J.-F., Semel, M. and Rees, D.E. (1992a) $A \& A, \mathbf{2 6 5}, 669$

Donati, J.-F., Brown, S.F., Semel, M., Rees, D.E., Dempsey, R.C., Matthews, J.M., Henry, G.W. and Hall, D.S. (1992b) $A \mathscr{E} A$, 265, 682 